\title{
46. Jahrestagung der RWGN in Bonn - Nuklearmedizin in NRW mit moleku- laren Diagnose- und Therapieverfahren für die Zukunft stark aufgestellt
}

Rund 220 Teilnehmer, vorrangig Nuklearmedizinerinnen und Nuklearmediziner aus NRW und deren Mitarbeiterinnen und Mitarbeiter, trafen sich am 29. und 30. November 2019 zur 46. Jahrestagung der Rheinisch-Westfälischen Gesellschaft für Nuklearmedizin (RWGN e.V.) im Wissenschaftszentrum in Bonn. Unter der Leitung der Tagungspräsidenten Prof. Dr. Holger Palmedo und Dr. Holger Brockmann (Nuklearmedizinische Abteilung der Gemeinschaftspraxis für Radiologie und Nuklearmedizin sowie Betreiber des PET/CT-Zentrum am Johanniterkrankenhaus in Bonn) diskutierten zahlreiche Fachvertreter sowohl der Unikliniken als auch der nuklearmedizinischen Krankenhausabteilungen und Praxen der Region über neueste Forschungsergebnisse des Fachgebiets, insbesondere aus dem Bereich der molekularen Diagnostik und Therapie und deren Umsetzung in der Patientenversorgung.

Am Freitag stellten die Vertreter der nordrhein-westfälischen Universitätsklinika ihre aktuellen Forschungsprojekte - von Schilddrüsenkarzinomstudien über KI bis hin zur Leberszintigrafie - vor. Der Samstag war traditionell von Fortbildungsbeiträgen geprägt. Hier gab es immer einen sehr schönen Wechsel zwischen Vorträgen von Klinikern und Nuklearmedizinern, um das gegenseitige Verständnis für die jeweils andere Fachdisziplin zu erhöhen. Exemplarisch sei hier zum Beispiel die Kombination aus Orthopäden und Nuklearmedizinern zur Bildgebung bei der TEP genannt. Aber auch Experten aus den Bereichen der Gynäkologie, Orthopädie und Urologie fanden ihren Gegenpart in kompetenten Nuklearmedizinern, sodass sich auch hier die Ver-

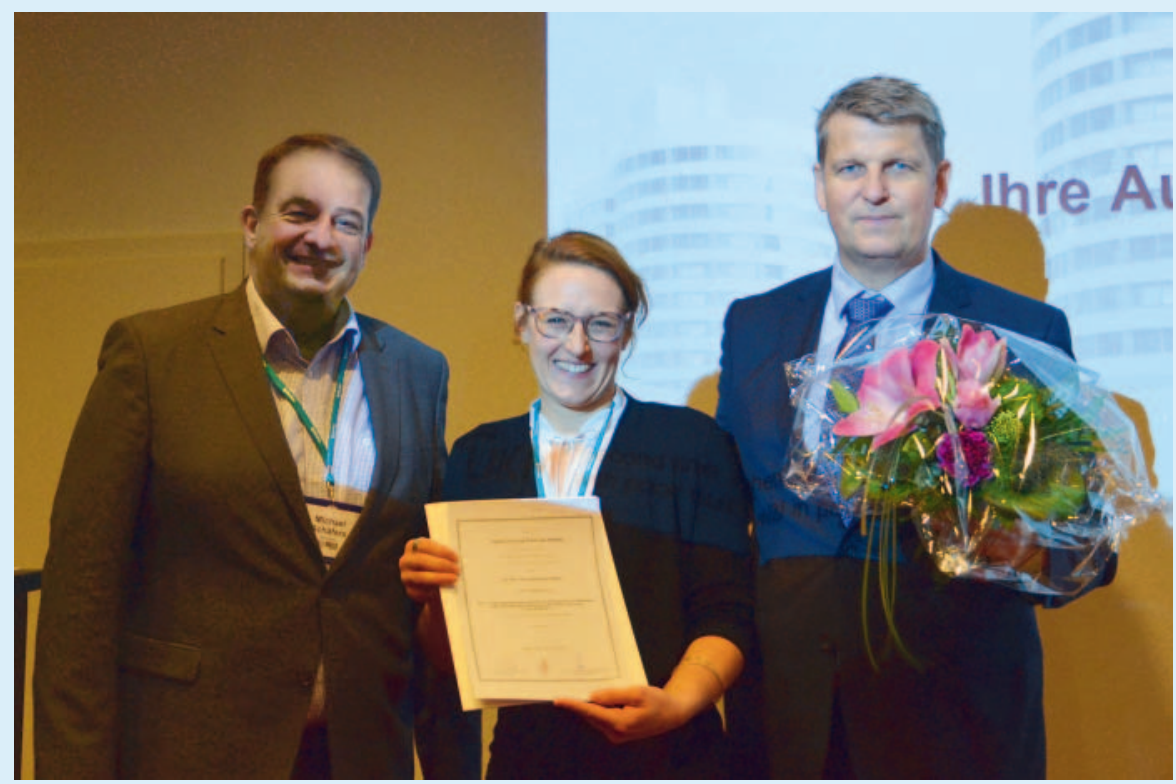

Preisverleihung Hans-Creutzig-Preis (Foto: Dr. Manuel Maria Weber). [rerif]

netzungen von klinischen Anforderungen und nuklearmedizinischen Antworten aufzeigten. Insgesamt wurde so noch einmal sehr schön dargestellt, dass die nuklearmedizinischen Untersuchungsmethoden und Therapien einen unverzichtbaren Bestandteil insbesondere auch im Work-up komplexerer Erkrankungen darstellen.

Zeitgleich zur ärztlichen Fortbildung lief am Samstagvormittag ein sehr gut besuchtes MTRA-Fortbildungsprogramm, welches ausgesuchte Themen vom FDG-PET/CT bis hin zur nuklearmedizinischen Diagnostik beim Nebenschilddrüsenadenom abdeckte.

Während der Veranstaltung erfolgten die Vergabe des Hans-Creutzig-Preises an Frau
Dr. rer. nat. Katharina Kessel aus dem Universitätsklinikum Münster sowie die Verleihung der Ehrenmitgliedschaften der RWGN an Herrn Prof. Dr. Hans-Jürgen Biersack aus Bonn und Herrn Prof. Dr. Harald Schicha aus Köln.

Die hohe Beteiligung zeigte die sehr gute Akzeptanz der langjährig etablierten Veranstaltung.

Der Vorsitzende der RWGN, Prof. Dr. Dr. Wolfgang Schäfer, äußerte sich zum Abschluss äußerst zufrieden über den Verlauf der diesjährigen Veranstaltung und dankte neben Professor Palmedo und Dr. Brockmann insbesondere auch der vokativ $\mathrm{GmbH}$ für das gelungene Veranstaltungsmanagement. 